Fußgesundheit

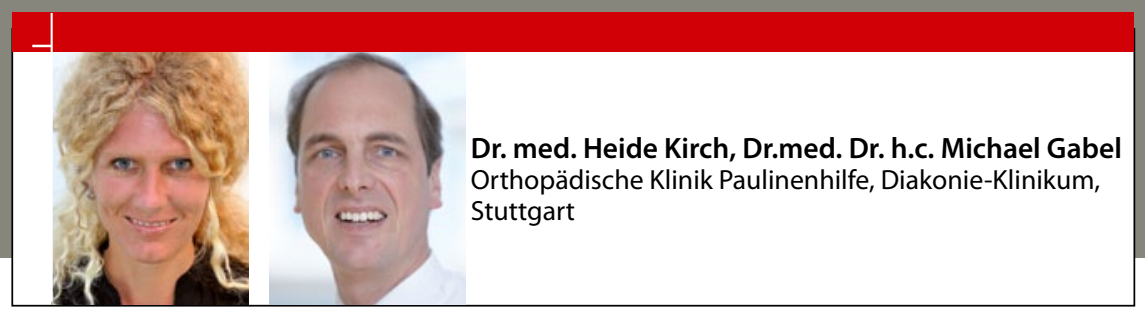

37- Hallux valgus

40 - Fersenschmerz

42 - Deformitäten der Kleinzehen

46 - Podologie

Plädoyer für ein neues Fußbewusstsein

\title{
Ein Wunderwerk aus 26 Knochen
}

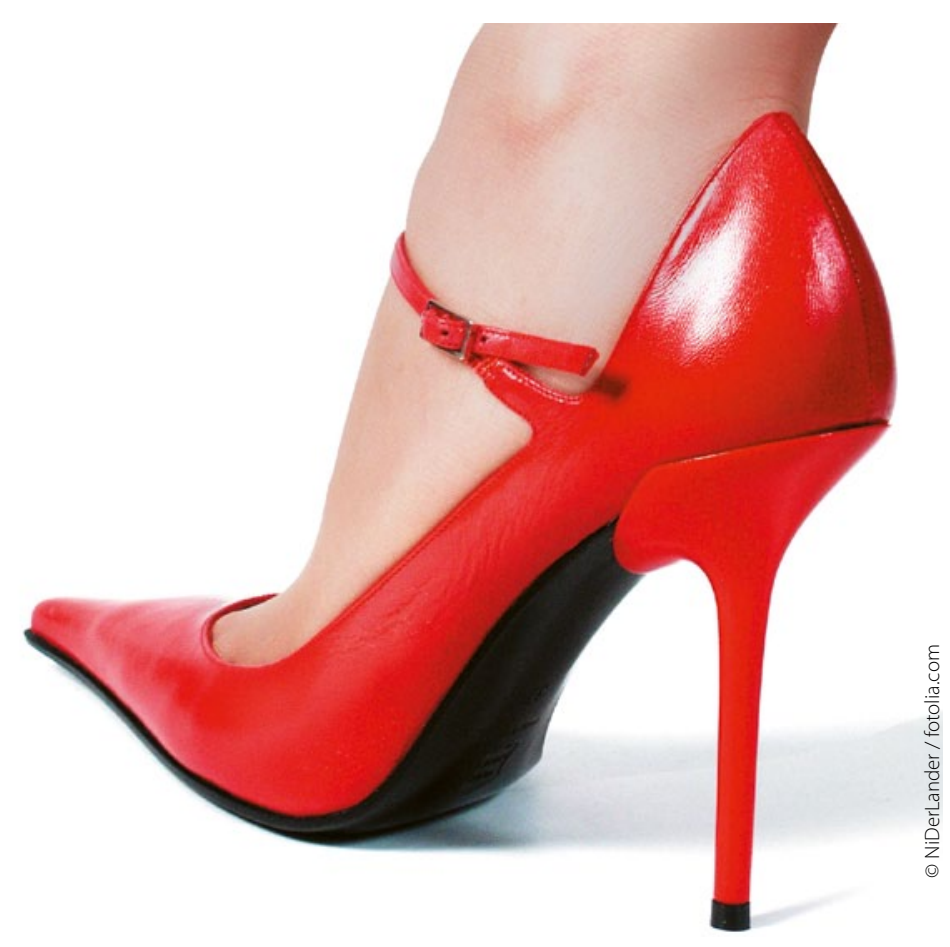

— "Man's foot is all his own. It is unlike any other foot. It is the most distinctly human part of the whole of his anatomical make-up ... We may, therefore, assume that we have any guarantee that the foot is a proper subject for examination for its own sake." (Frederic Wood Jones [1879-1954] in "Structure and Function as seen in the Foot", 1944).

Oftmals führen unsere Füße ein Schattendasein. Sie sind dem Boden am nächsten, tiefstehend, ordinär, unschön, werden mit Gleichgültigkeit gestraft. Häufig missachten wir sie und vergessen, wie hilflos wir ohne sie wären. Dabei sind sie das ausgereifteste Organ des Menschen. Erst wenn sie schmerzen, finden sie in unserer Gesellschaft Beachtung. Hiervon sind Ärzte nicht auszunehmen.

Seit den 80er-Jahren des vergangenen Jahrhunderts erfolgt nun ein Umdenken. Nach jahrelanger, zivilisationsbedingter Kasteiung mit ungeeignetem Schuhwerk, harten Böden und mangelnder Zuwendung sind wir wieder auf die Füße gekommen. Die Mode erlaubt das Tragen offener Schuhe und Barfußlaufen. Ein Zeigen nackter Füße ist nicht mehr verpönt. Man beschäftigt sich mit ihnen.

Bis vor gut 20 Jahren waren Fußspezialisten "Mangelware“. 1993 gründeten einige Fuß-Enthusiasten aus einem Arbeitskreis der Deutschen Gesellschaft für Orthopädie und Orthopädische Chirurgie die D.A.F., die Deutsche Assoziation für Fuß und Sprunggelenk. Man spezialisierte sich auf das Wunderwerk aus 26 Knochen, 33 Gelenken und über 100 Sehnen, Muskeln und Bändern, das uns im Laufe unseres Lebens Tausende von Kilometern trägt.

Das immer bessere Verständnis der Füße und der Fußpathologien und hinzugewonnene diagnostische Möglichkeiten führten zu einem stetigen Wandel der Therapiemöglichkeiten von Fußbeschwerden. Resezierende operative Eingriffe wurden im Verlauf immer häufiger durch gelenkerhaltende Operationen ersetzt.

Ein immer differenziertes Vorgehen unter besonderer Berücksichtigung prophylaktischer und konservativer Behandlungsmaßnahmen sowie die Wiedererlangung eines neuen „Fußbewusstseins" ermöglichen heute eine adäquate und zielgerichtete ganzheitliche Therapie. Ein ganzheitliches Behandlungskonzept setzt eine Synergie unterschiedlicher Professionen und Kompetenzen voraus, die eine weiter fortschreitende Spezialisierung der Medizin im Umkehrschluss impliziert.

Das Thema Fuß erfordert eine Zusammenarbeit von Fußspezialisten (Orthopäden, Unfallchirurgen), Diabetologen, Gefäßchirurgen, Orthopädie(schuh-)technikern, Podologen und Physiotherapeuten sowie dem eine Orientierungshilfe bietenden Hausarzt. Natürlich trägt insbesondere der mündige Patient selbst zum Behandlungserfolg oder -misserfolg bei. Adäquates Schuhwerk, die Wiedererlangung eines neuen „Fußbewusstseins" im Speziellen und eines natürlichen Körpergefühls im Allgemeinen können helfen, Fußbeschwerden vorzubeugen oder zu lindern.

Auf den folgenden Seiten bieten wir Beiträge zu dem Thema Fußgesundheit. Es erfolgt eine Fokussierung auf vier häufige Beschwerdebilder: Hallux valgus, Kleinzehendeformitäten, Fersenschmerz sowie Haut- und Nagelerkrankungen im Bereich des Fußes. 